Check for updates

Cite this: RSC Adv., 2019, 9, 21418

\title{
Anti-oxidative effects of catechins and theaflavins on glutamate-induced HT22 cell damage
}

\begin{abstract}
Jinting $\mathrm{He}$, (D) ${ }^{\text {a }}$ Lei $\mathrm{Xu}{ }^{\mathrm{a}}$ Le Yang ${ }^{\mathrm{b}}$ and Caixia Sun ${ }^{\star c}$
Background: Glutamate is an excitatory neurotransmitter that is involved in cell stress caused by oxidation. Polyphenolic compounds display various potential neuroprotective properties due to their ability to donate electrons and hydrogen atoms. Method: In this study, we evaluate the protective effect towards glutamateinduced HT22 cell damage. Two families of polyphenolic compounds are investigated, including the monomer polyphenol catechins, as well as the dimerized theaflavins. The cell apoptosis and intercellular ROS production are quantified by flow cytometry, and the protective mechanism is evaluated by quantifying the expression of cell apoptosis and energy related proteins. Result: Both sets of compounds protect cells against glutamate-induced oxidative stress, partially restore the cell viability, and prevent cells from apoptosis via bcl-2 and bax regulation, and attenuate intercellular ROS production. We demonstrate here that the protective effect is mediated by multiple factors, including reducing intracellular $\mathrm{Ca}^{2+}$ concentration, increasing glutathione level and related enzyme activity. Thus, the phosphorylation of AMP-activated protein kinase (AMPK) and extracellular signal-regulated kinase (ERK) show inverse correlation of activity after catechins and theaflavins stimulation. Conclusion: These results suggest both catechins and theaflavins compounds protect cells from glutamate-induced damage via cell apoptosis-related proteins and indirect regulation of cellular energy enzymes. These natural sourced antioxidants provide potential therapeutic agents for glutamate accumulation and toxicity related diseases.
\end{abstract}

Received 11th April 2019

Accepted 30th June 2019

DOI: 10.1039/c9ra02721a

rsc.li/rsc-advances
The antioxidant properties of polyphenolic compounds have been widely studied. They act as prevention agent to guard against the development of neuronal dysfunction and cellular death. ${ }^{22}$ Catechins, comprise epigallocatechin-3-gallate (EGCG), epigallocatechin (EGC), epicatechin-3-gallate (ECG), and epicatechin (EC), are major tea polyphenolic compounds. They are catalyzed to be oxidized and dimerized naturally occurring by oxidase enzymes (Fig. 1A and B). ${ }^{23}$ Theaflavins are typical dimerized product of catechins, and also the main pigments during tea fermentation. The chemical constitution of theaflavins is tightly dependent by substrate (Fig. 1B). There are four common dimerized compounds, named theaflavins (TF1), theaflavins-3- $o$-monogallate (TF2a), theaflavins-3'-o-monogallate (TF2b) and theaflavins-3,3'-o,o-digallate (TF3), all of which harbor more hydroxy groups in a single molecular. ${ }^{24}$ Due to the ability of hydroxyl to donate electrons and hydrogen atoms, we assume both families of catechins and theaflavins compounds potentially function as antioxidants. Although the biological effect of catechins were widely investigated, its dimerized compounds, theaflavins were rarely studied. In addition, those two families of polyphenolic compounds might reside on different targets inside the cells, since they are comprised by unique structure and different molecular size. ${ }^{25,26}$ Therefore, it is interesting to determine and compare the anti-oxidative effect of catechins and theaflavins. The protective effect of catechins, especially EGCG, has been widely investigated, which is involved in numerous
${ }^{a}$ Department of Neurology, China-Japan Union Hospital, Jilin University, Changchun, China, 130031

${ }^{b}$ People's Hospital of Jilin Province, Changchun, China, 130021

${ }^{c}$ Department of Gastrointestinal Colorectal and Anal Surgery, China-Japan Union Hospital, Jilin University, 126 Xiantai St, Erdao Qu, Changchun, Jilin, China, 130031.E-mail: cxsunjlu@163.com 
A<smiles>Oc1cc(O)c2c(c1)O[C@H](c1ccc(O)c(O)c1)[C@H](O)C2</smiles>

EC

B<smiles>O=c1cc(O)c2cc3c(O)c(O)cc([C@H]4Oc5cc(O)cc(O)c5C[C@H]4O)c3cc(C3Oc4cc(O)cc(O)c4CC3O)cc1-2</smiles>

$\mathrm{EC}+\mathrm{EGC}$

Theaflavins (TF1)<smiles>Oc1cc(O)c2c(c1)O[C@H](c1cc(O)c(O)c(O)c1)[C@H](O)C2</smiles>

EGC<smiles>O=C(OC1Cc2c(O)cc(O)cc2O[C@H]1c1cc(O)c(=O)c2c(O)c(O)cc([C@H]3Oc4cc(O)cc(O)c4C[C@H]3O)c2c1)c1cc(O)c(O)c(O)c1</smiles>

$\mathrm{ECG}+\mathrm{EGC}$<smiles>O=C(O[C@H]1Cc2c(O)cc(O)cc2OC1c1ccc(O)c(O)c1)c1cc(O)c(O)c(O)c1</smiles>

ECG

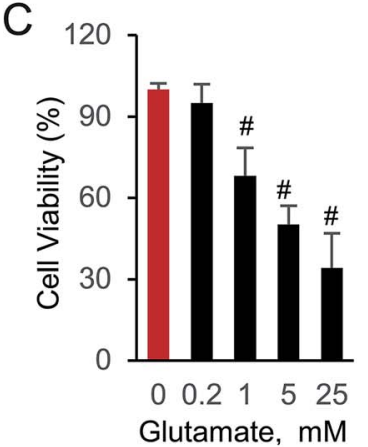

F

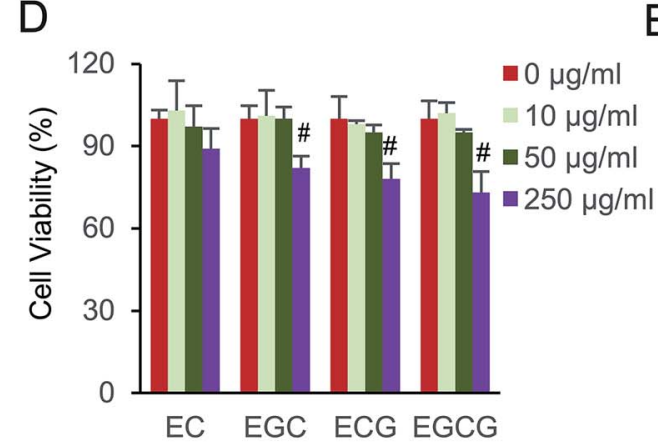<smiles></smiles>

(O)<smiles>O=C(OC1Cc2c(O)cc(O)cc2OC1c1cc(O)c(O)c(O)c1)c1cc(O)c(O)c(O)c1</smiles>

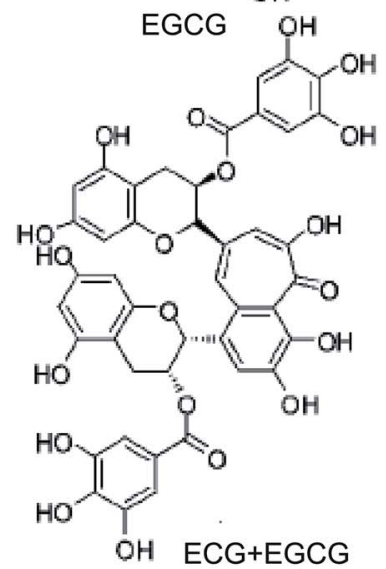

Theaflavins-3,3'-O,O-digallate(TF3)

E

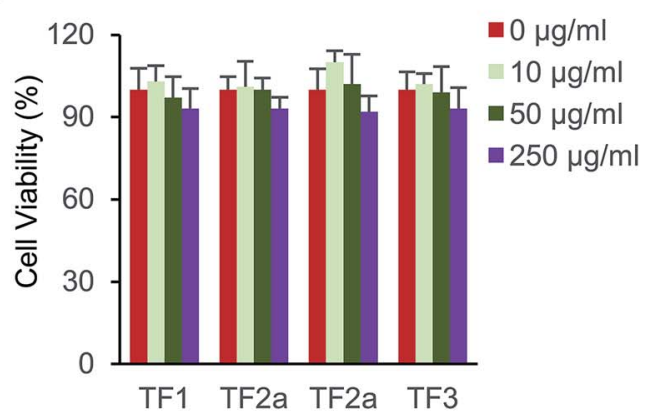

G

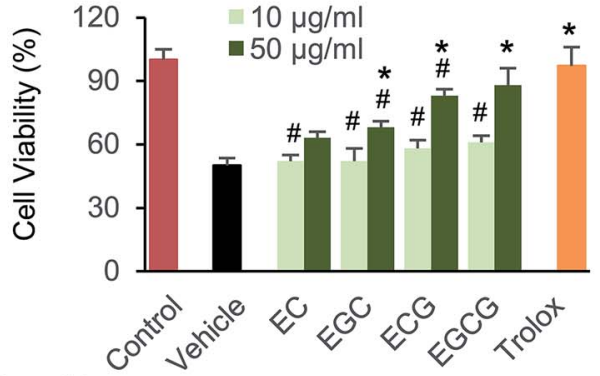

Catechins

Glutamate $(5 \mathrm{mM})-+++++$

Trolox $(50 \mu \mathrm{M}) \quad-\quad-\quad-\quad-\quad-\quad-\quad+$

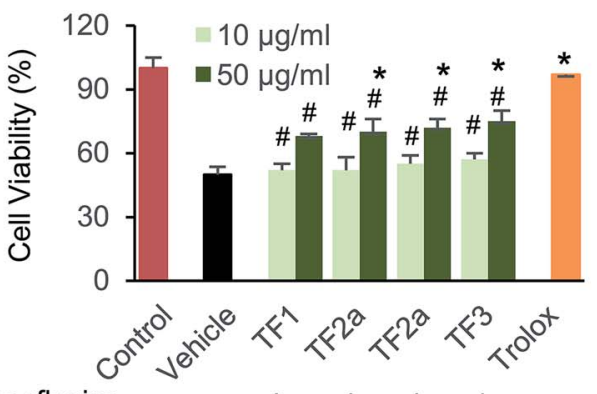

Theaflavins - -++++ -

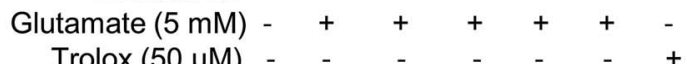

Fig. 1 Catechins and theaflavins restore glutamate-induced HT22 cell viability. (A) Chemical structure of catechins, including EC, EGC, ECG and EGCG. (B) Chemical structure of theaflavins, including TF1, TF2a, TF2b and TF3. (C) Glutamate (D) catechins and (E) theaflavins cell viability assay was done by $\mathrm{CCK}-8$ kit. (F) Protective effect of catechins and (G) theaflavins against glutamate-induced cell viability. Data were expressed as the mean $\pm \mathrm{SD}(n=6) .{ }^{\#} P<0.05$ vs. the control group, $* P<0.05$ vs. the vehicle group.

physiological activities such as anti-oxidant, anti-tumor, and antiinflammation. ${ }^{27-29}$ Glutamate-induced cell damage is also partially restored by those compounds. ${ }^{30-32}$ Multiple cellular signaling pathways are affected by glutamate-induced oxidative stress, including extracellular signal-regulated kinase (ERK), cJun N-terminal kinase (JNK) and p38. ${ }^{33,34}$ Notably, AMPactivated protein kinase (AMPK) is the most essential regulator, leading its function on cell proliferation, differentiation, and 
inflammation..$^{35,36}$ Although protective effect of catechins on oxidative stress has been reported before, little work on signaling pathway and regulator has been investigated on its dimerized product, theaflavins. Specifically, the function of theaflavins on CNS is seldom reported. Thus, the protective mechanism of catechins and theaflavins on glutamate-induced neural cell damage is still unclear.

We explored here the neuroprotective effect of catechins, theaflavins and how cell apoptosis and energy proteins were involved in glutamate-induced neurotoxicity in HT22 mouse hippocampal neuronal cell line. Pretreatment with either catechins or theaflavins, restored the cell viability from glutamate toxicity. Trolox, a synthesized vitamin E derivative, was used as positive control. When cells were supplied with high concentration glutamate, both cell apoptosis and necrosis were induced. The two typical representative compounds, EGCG and TF3, decreased glutamateinduced apoptosis and ROS production, of which, EGCG appeared to be a litter more effective. Intercellular $\mathrm{Ca}^{2+}$ influx, mitochondrial dysfunction and depletion of antioxidant defense enzyme, glutathione, contributed to the excessive ROS production. EGCG and TF3 both contributed to antioxidant activity, as well as neuroprotective ability against glutamate. Further, both EGCG and TF3 activated the phosphorylation of AMPK, but reduced phosphorylation ERK, which indicated the protective effect was caused by the residue, but not the entire structure of those compounds. Overall, these data suggested that both catechins and theaflavins protected glutamate-induced HT22 cell damage.

\section{Materials and methods}

\subsection{Materials and regents}

Dulbecco's modified Eagle medium (DMEM) and fetal bovine serum (FBS) were purchased from GIBCO Life Technologies. HT-22, mouse hippocampal neuronal cell line, was purchased from Fuxiang Biotech, China. Cell Counting Assay Kit-8 (CCK-8) was produced by Gold Biotechnology, China. Fluorescein isothiocyanate-conjugated Annexin V (Annexin V-FITC) and propidium iodide (PI) Detection Kit was purchased from BD Biosciences. The first antibodies P-AMPK Thr172 and AMPK $\alpha$, P-ERK1/2 and ERK1/2 came from Cell Signaling Technology, Bcl-2 and Bax were purchased from Santa Cruz Biotechnology. While actin and the secondary horseradish peroxidase-coupled antibody, donkey anti-rabbit IgG, goat anti-mouse IgG were all from Sigma-Aldrich. All the florescence dyes, including fura3AM, rhodamine $123,5^{\prime}, 5^{\prime}$-dithiobis 2-nitrobenzoic acid, and nicotinamide adenine dinucleotide phosphate were purchased from molecular probe. Four tea catechins, including EC, EGC, ECG, EGCG, and four theaflavins, TF1, TF2a, TF2b and TF3 were purchased from Sigma-Aldrich. The chemical structures of the investigated compounds in this study are shown in Fig. 1A and B. All other chemicals made in China were of analytical grade.

\subsection{Cell culture and viability assay}

HT22 cells were routinely cultured in DMEM medium supplied with $10 \%$ FBS $(\mathrm{v} / \mathrm{v})$, and double antibiotics $100 \mathrm{U} \mathrm{mL}^{-1}$ penicillin, $100 \mu \mathrm{g} \mathrm{mL}{ }^{-1}$ streptomycin were added into the medium.
Cells were incubated in a humidified atmosphere at $37{ }^{\circ} \mathrm{C}$ containing $5 \% \mathrm{CO}_{2}$. Replaced the medium every other day to maintain regular cell growth. All the experiment were performed when cell density reached roughly $70 \%$ area.

Cell viability was measured using the CCK- 8 kit, followed by the manufacturer's instruction. Briefly, 10000 cells were seeded into the 96-well plates with the volume of $100 \mu \mathrm{L}$ for each well. After incubation for 24 hours, 10 or $50 \mu \mathrm{g} \mathrm{mL}^{-1}$ catechins and theaflavins, or $50 \mu \mathrm{M}$ trolox was added to cells for 12 hours. After that, $5 \mathrm{mM}$ glutamate was supplied for additional 3 hours. Cells were incubated with $10 \mu \mathrm{L}$ CCK-8 solution for 3 hours at $37{ }^{\circ} \mathrm{C}$. Finally, the absorbance at $450 \mathrm{~nm}$ in each well was measured using Microplate Reader (TECAN 10M).

\subsection{Flow cytometry analysis of apoptosis, necrosis and ROS production}

The HT22 cells were seeded into 6-well plate at 4 he $10^{5}$ cells per well for 24 hours. When appropriate, $50 \mu \mathrm{g} \mathrm{mL}{ }^{-1}$ EGCG, TF3, or $50 \mu \mathrm{M}$ trolox was added to cells and incubated 12 hours, and then $5 \mathrm{mM}$ glutamate was supplied for another 3 hours. The experiment detail was performed as before. ${ }^{24,37}$ Cell apoptosis and necrosis were evaluated by measuring the exposure of phosphatidylserine on cell membrane and permeable of DNA fluorescent dye. Cell pellets were resuspended and stained for 15 minutes in the buffer containing PI and Annexin V-FITC at room temperature in dark. The ROS production was quantified using 2,7-dichlorofluorescein diacetate (DCFDA). Cells were treated with $1 \mu \mathrm{M}$ DCFDA at $37{ }^{\circ} \mathrm{C}$ for 30 minutes. After stating, cells were passed through a $40 \mu \mathrm{m}$ cell strainer and then subjected to flow cytometry equipped with the Cell Quest software, at least 10000 cells were analyzed in all cases.

\subsection{Glutamate-induced physiology changes assay}

HT22 cells were plated in 48-well plates and incubated for 24 hours. After incubation, 10 and $50 \mu \mathrm{g} \mathrm{mL}{ }^{-1}$ of catechins or theaflavins, $50 \mu \mathrm{M}$ trolox was added to cells for 12 hours and then $5 \mathrm{mM}$ glutamate were added for 3 hours. The physiology changes assay was done as described with modifications. ${ }^{\mathbf{3 8 - 4 0}}$ Intracellular calcium $\left(\mathrm{Ca}^{2+}\right)$ concentration measurement were carried out using Fura3AM, a widely used calcium fluorescent ester indicator. After staining, cells were washed with buffer containing $20 \mathrm{mM}$ HEPES $\mathrm{pH} 7.0$ and $150 \mathrm{mM} \mathrm{NaCl}$. The $\mathrm{Ca}^{2+}$ concentration was determined by measuring fluorescence intensity at an excitation wavelength of 340 and $380 \mathrm{~nm}$ and emission wavelength of $500 \mathrm{~nm}$.

The fluorescent dye, rhodamine 123 (Rho123) was used to determine mitochondrial membrane potential $(\Delta \Psi)$. Cells were stained with Rho123 for $15 \mathrm{~min}$ at $37^{\circ} \mathrm{C}$ and followed by a quick wash to remove excess dye. The Rho123 concentration was quantified by fluorescence intensity at an excitation wavelength of $488 \mathrm{~nm}$ and emission wavelength of $520 \mathrm{~nm}$.

The glutathione level, reductase and peroxidase assays were performed. Briefly, the glutathione was measured based on the reduction of $5^{\prime}, 5^{\prime}$-dithiobis 2-nitrobenzoic acid (DTNB) with reductase and nicotinamide adenine dinucleotide phosphate $(\mathrm{NADPH}) .{ }^{41}$ Cells were lysed with sulfosalicylic acid. Centrifuged 
the cell lyses at $4000 \mathrm{rpm}$ for $30 \mathrm{~min}$ at $4{ }^{\circ} \mathrm{C}$. Supernatant was separated and added to final 5 units per $\mathrm{mL}$ glutathione disulfide reductase, $0.3 \mathrm{mM} \mathrm{NADPH}$ and $0.5 \mathrm{mM}$ DTNB. The absorbance was quantified at the wavelength $412 \mathrm{~nm}$. Glutathione reductase was measured by monitoring the reduction of oxidized glutathione in the presence of NADPH. Glutathione peroxidase activity was calculated by quantifying the rate of oxidation of GSH to GSSG. The decrease absorbance was quantified at $340 \mathrm{~nm}$.

\subsection{Western blot assay}

The HT22 cells were lysed by buffer ( $20 \mathrm{mM}$ Tris-HCl pH 7.0, $1 \mathrm{mM}$ EDTA, $50 \mathrm{mM} \mathrm{NaCl}, 25 \mathrm{mM} \mathrm{NaF}, 2 \mathrm{mM}$ orthovanadate, $1 \%$ Triton X 100 and a protease inhibitor cocktail). Centrifuged the cell lysate at $12000 \mathrm{rpm}$ for 30 minutes at $4{ }^{\circ} \mathrm{C}$. The supernatant was subjected to protein concentration assay by Bradford methods. ${ }^{42}$ Equal amount of $100 \mu \mathrm{g}$ protein was loaded to SDSPAGE and then transferred to nitrocellulose membrane as did before. ${ }^{43}$ The nitrocellulose membrane was blocked in $5 \%$ nonfat milk for at least 1 hour, and incubated with primary antibodies overnight. The next day, the membrane was washed three times in PBS. A horseradish peroxidase-conjugated second antibody was added and the membrane was incubated 1 hour, followed by ECL chemoluminescence system. The intensity of the signals was semiquantified by ImageJ.

\subsection{Statistical analysis}

All the experiments were performed for at least three biological repeats. The result was presented as average \pm standard deviation (SD). Statistical analysis was performed by SPSS software version 21.0 (IBM Corp., Armonk, NY, USA). One-way ANOVA was used to evaluate significant difference. Tukey's test was used as the post hoc test between different samples. $P<0.05$ was used to statistically evaluate significant. The symbol \# indicated $P<0.05$ compared to control group, and * shown $P<0.05$ compared to vehicle group.

\section{Results}

\subsection{Neuroprotective effect of catechins and theaflavins on glutamate-induced cell viability}

Catechins, including (-)-epicatechin (EC), (-)-epicatechin gallate (ECG), (-)-epigallocatechin (EGC) and (-)-epigallocatechin gallate (EGCG), are monomeric polyphenolic compounds. While,
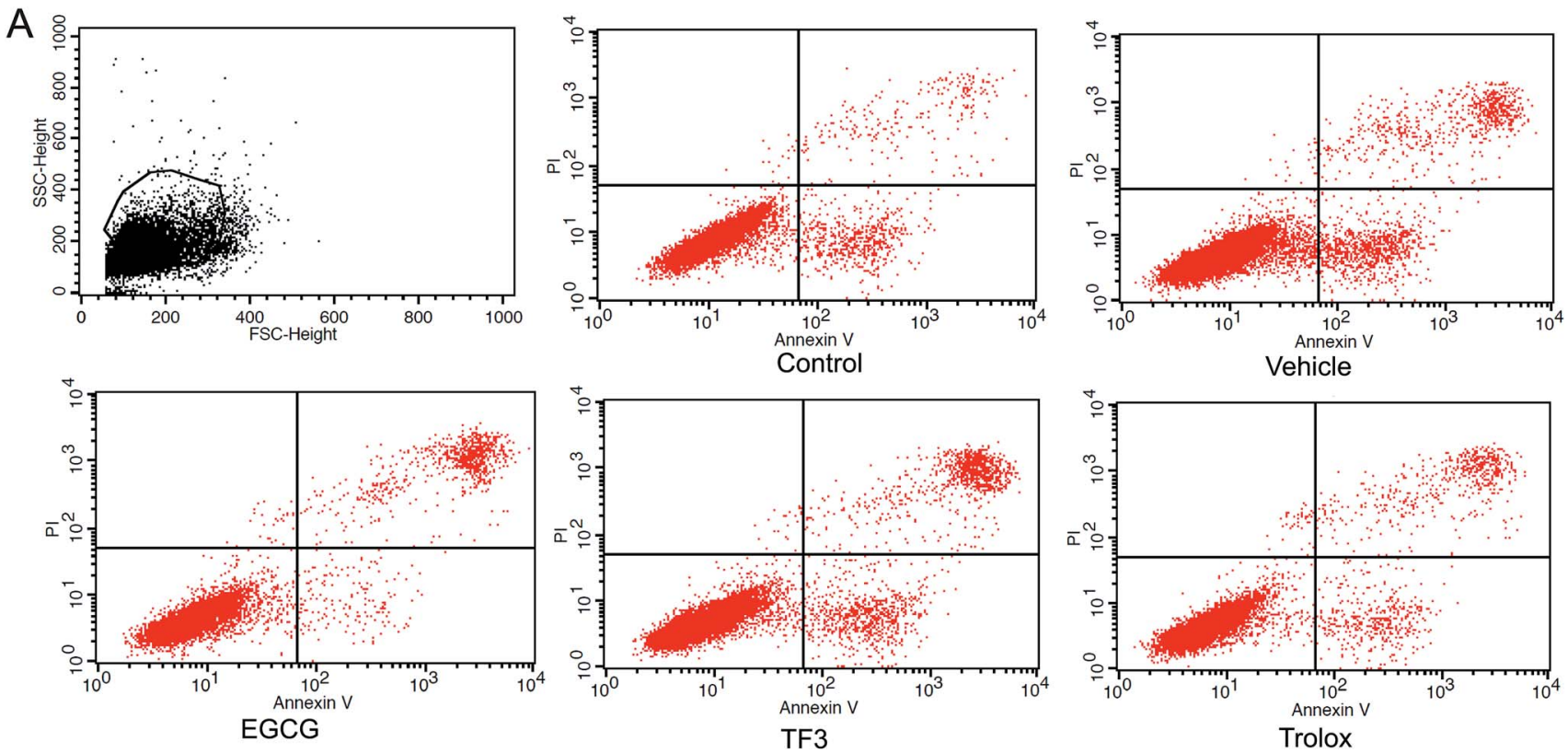

B

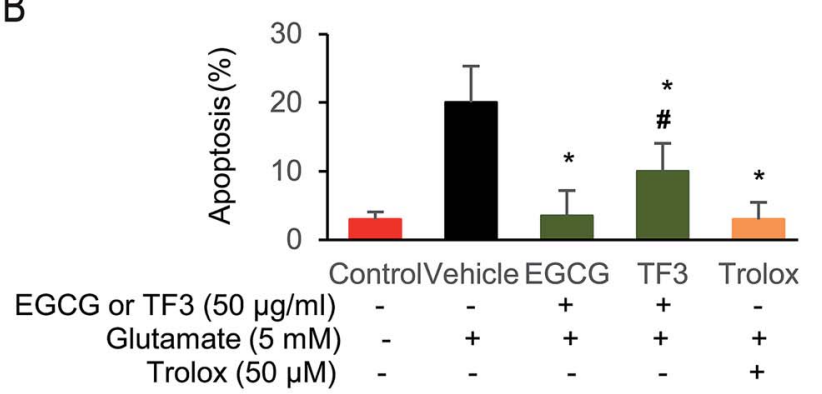

C

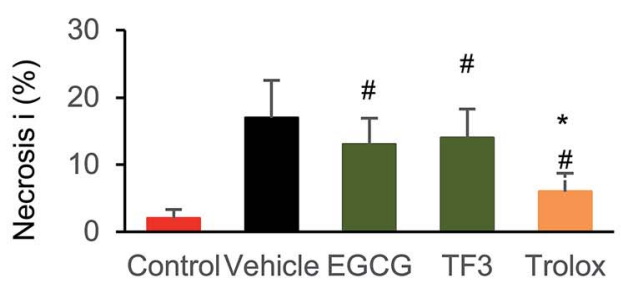

Fig. 2 EGCG and TF3 attenuated glutamate-induced apoptosis and necrosis in HT22 cells. (A) Necrosis and apoptosis cells were stained by Annexin V FITC and PI and determined by flow cytometry ( $n=10000$ cells). (B) Quantification of apoptosis cells. (C) Quantification of necrosis cells. ${ }^{\#} P<0.05$ vs. the control group, ${ }^{*} P<0.05$ vs. the vehicle group. 
oxidized and dimerized of two catechins, will generate theaflavins (TF), including theaflavins (TF1), theaflavins-3-o-gallate (TF2a), theaflavins- $3^{\prime}$ - $o$-gallate (TF2b) and theaflavins-3, $3^{\prime}$-o,o-digallate (TF3). We first determined how exogenous glutamate, catechins and theaflavins affected cell viability. The data indicated that the cell viability was significantly decreased by glutamate in a dosedependent manner (Fig. 1C). The cell viability was about 50\% when $5 \mathrm{mM}$ glutamate was added. If longer incubation time is used, maybe less glutamate should be added to maintain 50\% cell viability. We prefer to use $5 \mathrm{mM}$ glutamate, which is higher than intracellular glutamate concentration. Therefore, HT22 cells incubated for 3 hours with $5 \mathrm{mM}$ glutamate was used to induce cell damage for the following experiment. When adding 10 and $50 \mu \mathrm{g} \mathrm{mL}{ }^{-1}$ of tested compounds, no significant cell viability change was found compared with cells without any compound (Fig. 1D and E). Next, we evaluated the neuroprotective effect of those compounds on glutamate-induced cell viability in HT22 cells (Fig. 1F and G). When cells were supplied with catechins, EGCG was the most effective compound to protect the cells against glutamate toxic. While, the four theaflavins restored the cell viability at almost equal level. $50 \mu \mathrm{M}$ trolox, the positive control here, also significantly protected HT22 cells against glutamate-induced neurotoxicity. The results indicated that both catechins and theaflavins significantly increased viability of glutamate exposed HT22 cells.

\subsection{EGCG attenuated glutamate-induced HT22 cell apoptosis}

It has been previously reported that EGCG leads to a protection effect in apoptosis and necrosis in primary astrocytes. ${ }^{\mathbf{4 4}}$ However, the comparison between catechins and its oxidized products theaflavins has not been investigated yet. To this end, we selected the most effect catechins EGCG and one of the dimerized compound TF3, determined how those compounds prevented cell from apoptosis or necrosis. We took advantage of the flow cytometry method by double staining Annexin V and PI to distinguish those two events. When glutamate was added in the vehicle group, both necrosis and apoptosis cells were produced (Fig. 2A-C). EGCG and trolox significantly reduced cell apoptosis to $3.1 \%$ and $3.5 \%$ compared with vehicle group ( $p$ $<0.05)$. However, TF3 was less effective, and generated $10.1 \%$ apoptosis cells $(p<0.05)$. The necrosis could not be restored when either EGCG or TF3 was supplied. However, trolox pretreatment reduced cell necrosis significantly $(p<0.05)$. The data indicated EGCG and TF3 could not rescue glutamateinduced necrosis, but were effective on reducing cell apoptosis.

\subsection{Attenuated glutamate-induced ROS production of EGCG}

To investigate the involvement of oxygen radicals in glutamateinduced toxicity in HT22 cells, the accumulation of ROS was
A
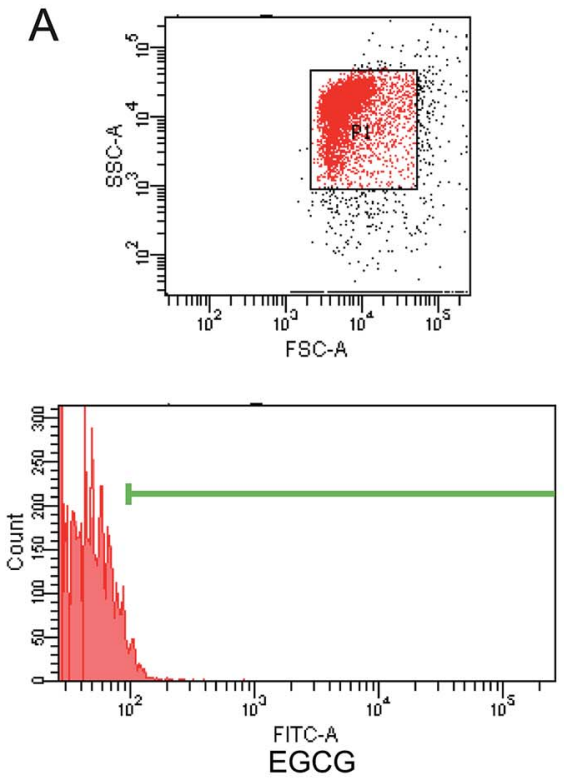
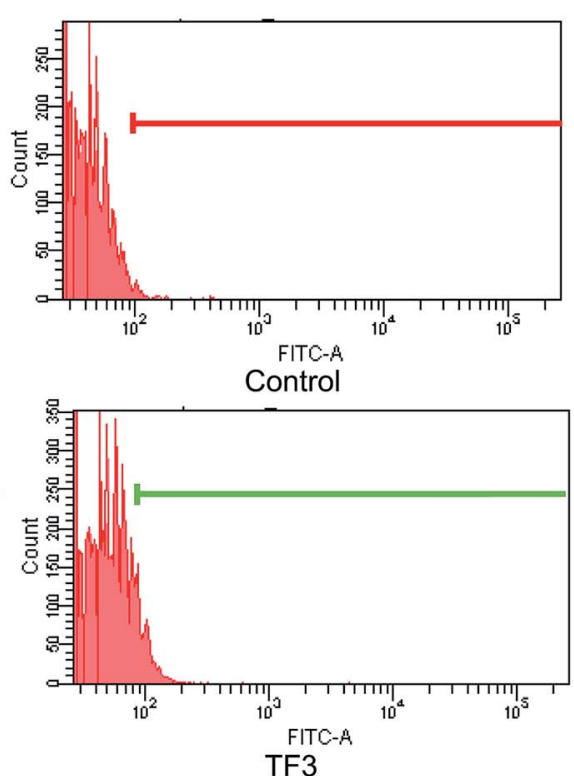

TF3
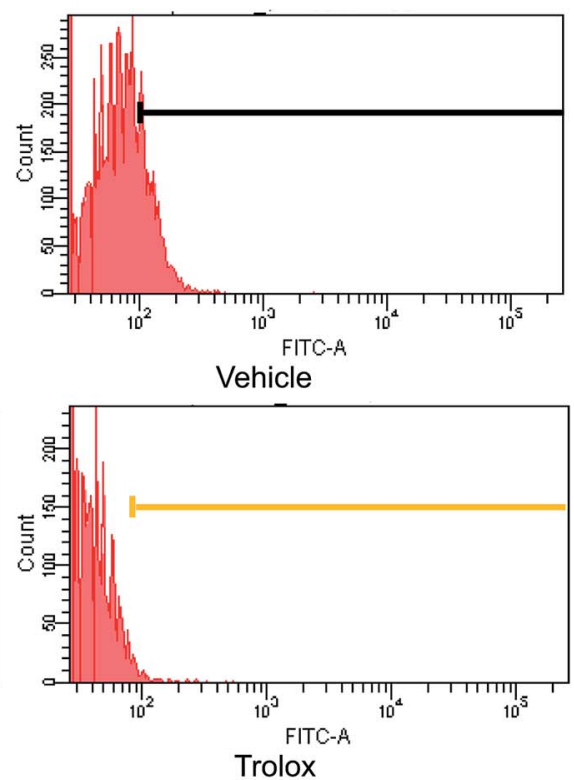

B

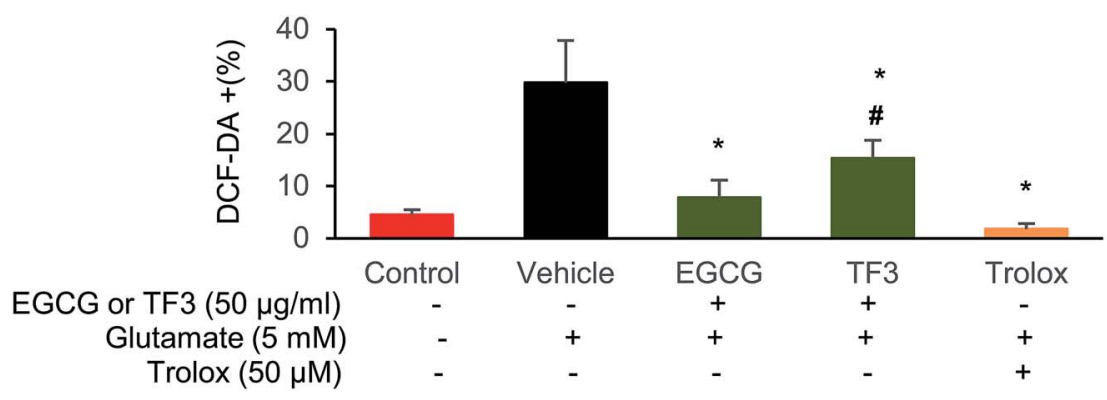

Fig. 3 EGCG and TF3 attenuated glutamate-induced ROS production in HT22 cells. (A) ROS production was measured using ROS-sensitive fluorometric probe DCFDA by flow cytometry ( $n=10000$ cells). (B) Quantification of ROS production. ${ }^{\#} P<0.05$ vs. the control group, ${ }^{*} P<0.05$ vs. the vehicle group. 
measured utilizing a converting reaction of DCFH-DA to DCF. ${ }^{45}$ Glutamate treatment significantly increased the ROS level to $29.8 \%$ in HT22 cells compared to that $4.5 \%$ in the control group (Fig. 3A and B). When HT22 cells were added with EGCG or TF3, the relative ROS positive cells were $7.8 \%$ and $15.3 \%$, respectively, which is significantly lower than the vehicle group ( $p<$ 0.05). The positive control in trolox group, the increased ROS was even clearly suppressed $(p<0.05)$. The results suggested the inhibition effects of EGCG on accumulation of intracellular ROS was better than TF3.

\subsection{Glutamate excitotoxicity of catechins and theaflavins}

Next, the effect of glutamate exposure on intracellular $\mathrm{Ca}^{2+}$ levels in HT22 cells was examined (Fig. 4A). Intracellular $\mathrm{Ca}^{2+}$ was significantly increased when cells were supplied with $5 \mathrm{mM}$ glutamate from basal levels 278 to $330 \mathrm{nmol} \mathrm{L}^{-1}$. EC, ECG, EGC, and EGCG partially blocked the increased intracellular $\mathrm{Ca}^{2+}$ concentration, compared to vehicle group $(p<0.05)$. Notably, when $50 \mu \mathrm{g} \mathrm{mL}^{-1}$ EGCG was supplied, the intracellular $\mathrm{Ca}^{2+}$ concentration dropped to its minimum level at $280 \mathrm{nmol} \mathrm{L}^{-1}$. The intracellular $\mathrm{Ca}^{2+}$ remained almost unchanged when $10 \mu \mathrm{g}$ $\mathrm{mL}^{-1}$ of EC was used. In addition, TF1, TF2a, TF2b and TF3 treatment all significantly inhibited the intracellular $\mathrm{Ca}^{2+}(p<$ 0.05). $50 \mu \mathrm{g} \mathrm{mL}^{-1} \mathrm{TF} 3$ block the lowest $\mathrm{Ca}^{2+}$ concentration among the four theaflavins compounds.

We further investigated the protective effect of catechins and theaflavins on glutamate-induced mitochondrial membrane potential (Fig. 4B). When cells were supplied with $5 \mathrm{mM}$ glutamate, a decreased mitochondrial membrane potential was found, which was relative to $80.3 \%$ compared with control

\section{A}
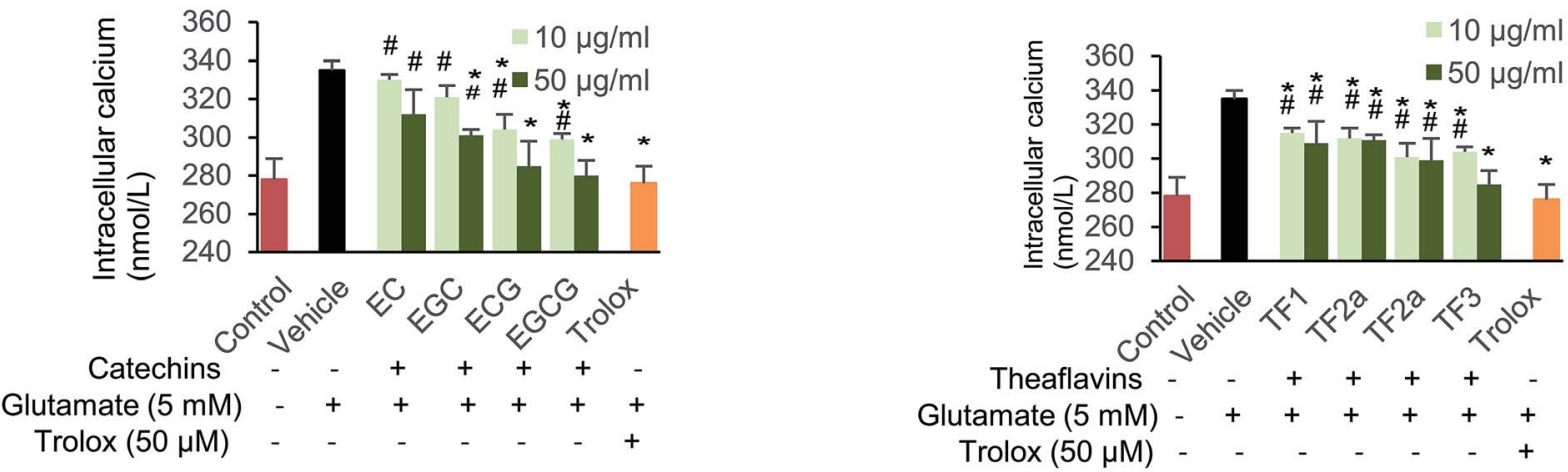

B
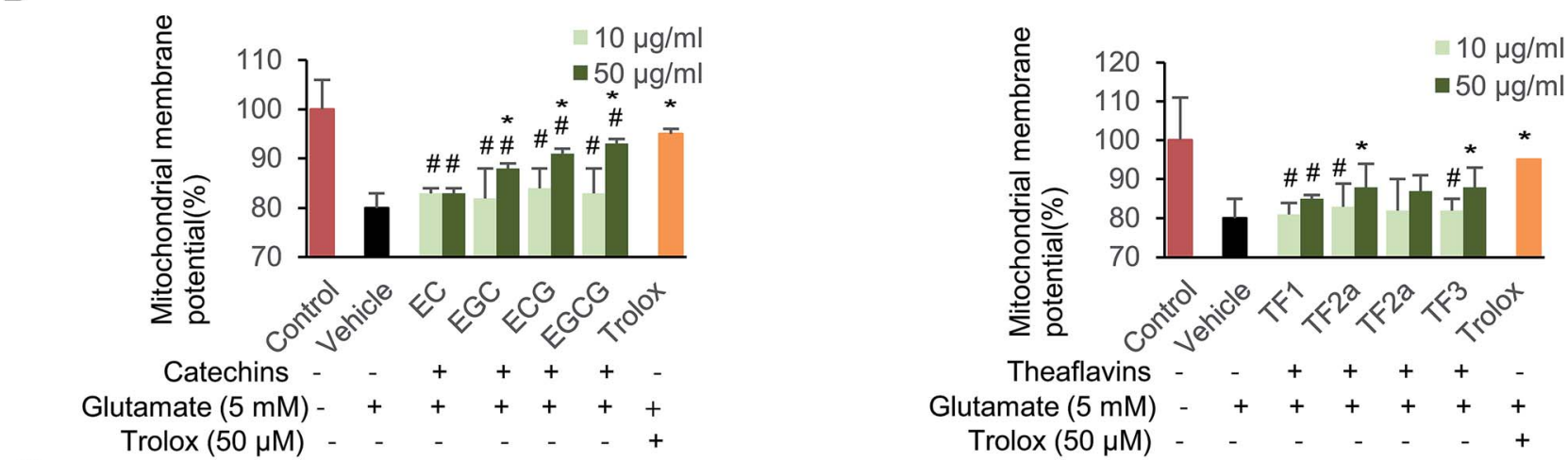

C

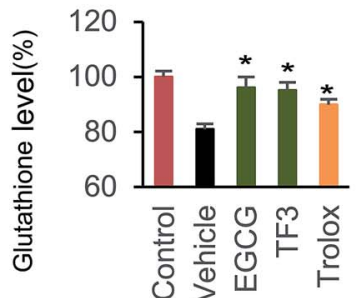

EGCG or TF3 $(50 \mu \mathrm{g} / \mathrm{ml})-\quad++$

Glutamate $(5 \mathrm{mM})-++++$

Trolox $(50 \mu \mathrm{M})-{ }_{-}-+$

EGCG or TF3 $(50 \mu \mathrm{g} / \mathrm{ml})--++$

Glutamate $(5 \mathrm{mM})-++++$

Trolox $(50 \mu \mathrm{M})$ - - - +

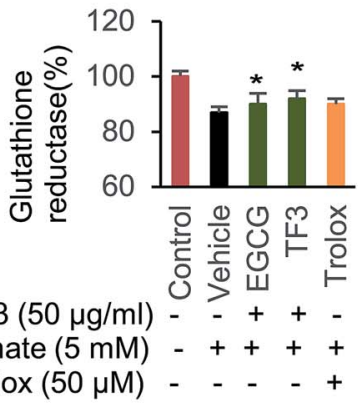

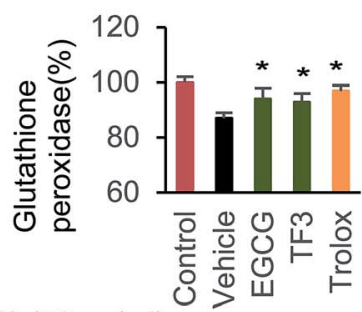

EGCG or TF3 $(50 \mu \mathrm{g} / \mathrm{ml})--++$

Glutamate $(5 \mathrm{mM})-++++$

Trolox $(50 \mu \mathrm{M})-\cdots+$

Fig. 4 Effect of catechins and theaflavins on glutamate-induced HT22 cell damage. (A) Changes of intercellular concentration of $\mathrm{Ca}^{2+}$. (B) Disruption of mitochondrial membrane potential. (C) Glutathione, glutathione reductase and glutathione peroxidase levels in $\mathrm{HT} 22$ cells. Data are presented as means \pm SD $(n=3)$. ${ }^{\#} P<0.05$ vs. the control group, ${ }^{*} P<0.05$ vs. the vehicle group. 
A

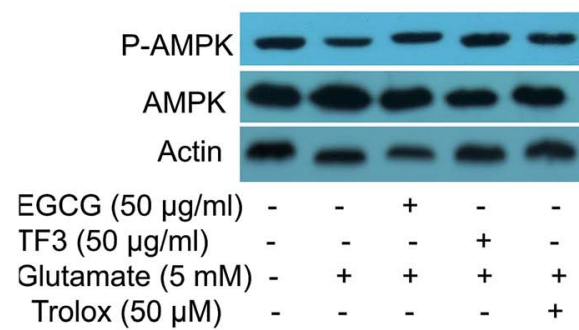

B

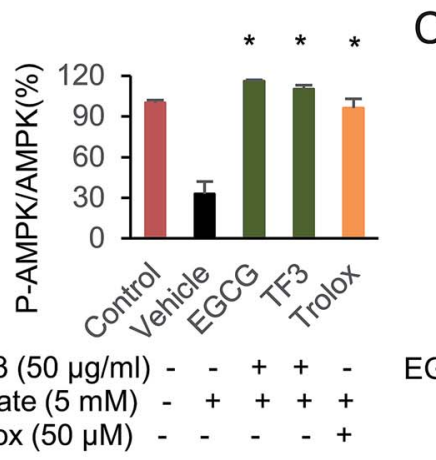

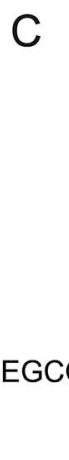

$\mathrm{F}$

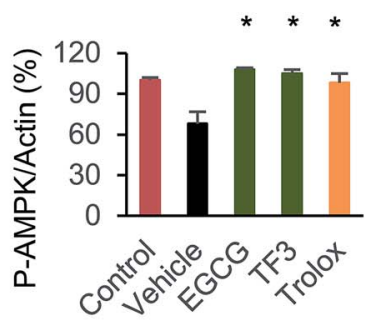

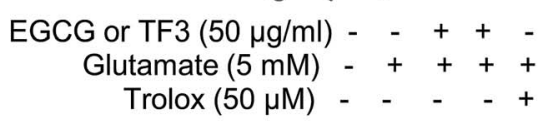

E

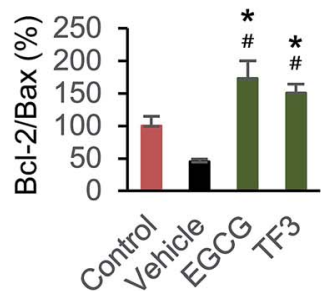

EGCG or TF3 $(50 \mu \mathrm{g} / \mathrm{ml})-\quad-\quad++$ Glutamate $(5 * \mathrm{mM})-+++$

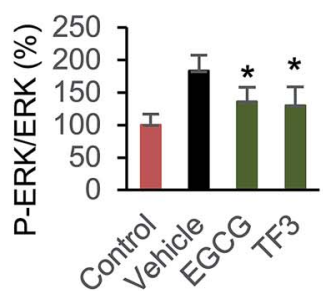

EGCG or TF3 $(50 \mu \mathrm{g} / \mathrm{ml})-\quad+\quad+$ Glutamate $(5 \mathrm{mM})-+++$

Fig. 5 Attenuating glutamate-induced HT22 cell damage was involved in AMPK activation. (A) Polyphenols protective effect on glutamateinduced p-AMPK protein expression. (B) Quantification of p-AMPK/AMPK by membrane intensity. (C) Quantification of p-AMPK/Actin by membrane intensity. (D) Polyphenols protective effect on glutamate-induced $\mathrm{p}-\mathrm{ERK}, \mathrm{Bax}$ and $\mathrm{Bcl}-2$ protein expression. (E) Quantification of Bcl2/Bax by membrane intensity. (F) Quantification of $p$-ERK/ERK by membrane intensity. ${ }^{*} P<0.05$ vs. the control group, ${ }^{*} P<0.05$ vs. the vehicle group.

group. The mitochondrial membrane potential increased when cells were treated by $50 \mu \mathrm{g} \mathrm{mL} \mathrm{m}^{-1}$ of EGC, ECG, and EGCG to $88.1 \%, 91.2 \%$ and $93.2 \%$, respectively $(p<0.05)$. While, $10 \mu \mathrm{g}$ $\mathrm{mL}^{-1}$ of catechins had little effect on restoring mitochondrial membrane potential. TF2a, TF2b and TF3 groups partially restored mitochondrial membrane potential $(p<0.05)$ as well.

Finally, we evaluated the effect of the investigated compounds on glutathione level, glutathione reductase and glutathione peroxidase activity on HT22 cells (Fig. 4C). Both EGCG and TF3 restore glutathione level $(p<0.05)$. Glutamate-induced glutathione reductase could be abolished by adding EGCG or TF3 $(p<$ $0.05)$. Glutathione peroxidase activity was partially complemented when cells were supplied with EGCG, TF3 or trolox ( $p$ $<0.05$ ). Taken together, exposure HT22 cells with $5 \mathrm{mM}$ glutamate, resulted in mitochondrial dysfunction and depletion of antioxidant defense systems, both catechins and theaflavins families compounds partially rescue the physiology.

\subsection{Protection of EGCG and TF3 on glutamate-induced cell damage was involved in apoptosis-related proteins, phosphorylation of AMPK and ERK}

The signal pathway involved in glutamate-induced HT22 cell damage has been investigate. ${ }^{46}$ However, the relationship between catechins, theaflavins and AMPK activation was not determined before. We performed the Western blot experiment here, and the results indicated a reduction of phosphorylated
AMPK at Thr172 when HT22 cells was treated with glutamate (Fig. 5A-C). EGCG or TF3 restored the phosphorylation to normal level compared with vehicle group $(p<0.05)$. Next, we found ERK pathway was involved in the suppression of AMPK activity. The results indicated an inverse correlation between the intensity of ERK phosphorylation and the degree of AMPK activation (Fig. 5B and F). Several apoptosis-related proteins, including Bcl-2 and Bax, were tested as well. The results clearly suggested that both catechins and theaflavins activated the ratio of bcl-2/bax, which precipitate to prevent apoptosis (Fig. 5E). Taken together, the data suggested that phosphorylated of AMPK and ERK was involved in protecting glutamateinduced HT22 cell damage. Cell apoptosis proteins were also activated in those process. EGCG and TF3 behaved almost equally in regulating these cell signal pathways.

\section{Discussion}

Catechins is a family of polyphenol in green tea. The antioxidative property and pathway regulation have been reported. ${ }^{47,48}$ However, few study has demonstrated protective effect of theaflavins family compounds against glutamateinduced neuronal damage. A remarkable observation in our study shown that excessive glutamate stimulation on HT22 cells leading multiple damage. Both catechins and theaflavins effetely protected the cells and accommodated cells to minimize glutamate damage. On the other hand, the chemical 
constitution and molecular size are different between catechins and theaflavins. The two families of polyphenolic compounds, activated AMPK phosphorylation, but negatively regulated ERK phosphorylation, which appears to play a key role in neuronal cell protection. Thus, the protective action was mainly due to antioxidative effect, since EGCG and TF3 significantly restored ROS production. Notably, ROS accumulation ultimately contribute to cell death in stroke, trauma as well as many other neurodegenerative disorders. ${ }^{49,50}$ This study first provided evidence that theaflavins and its derivate can also exert a neuroprotective effect on glutamate excitotoxicity.

Theaflavins are usually present in black tea, which are formed by the enzymatic oxidation, followed by condensation. ${ }^{24}$ They are the most important reddish-orange pigments and chemically well characterized. Because of its natural properties, it has the advantage of less cytotoxic to many cells. All theaflavins compounds are catechin dimers with a characteristic benzotropolone moiety, which is produced by a pair of quinones derived from dihydroxy and trihydroxy ${ }^{51}$ (Fig. 1A). The complex structure and multiple hydroxyl, lead to the strong enzyme inhibitions of theaflavins, as well as their anti-oxidative stress activities. Chemical relationships between catechins and theaflavins, are important for the viewpoint of the neural protective activities. EGCG and TF3, behave effectively on rescuing cell viability (Fig. 1C). EGCG directly interacts with proteins and phospholipids in the plasma membrane and regulates signal transduction pathways. ${ }^{52}$ Interestingly, mitochondrial dysfunction and depletion of antioxidant defense systems are also activated (Fig. 4A-C). EGCG and TF3 restore glutathione peroxidase and glutathione reductase level, in which the activities are partially responsible for susceptibility of cells to oxidative stress. ${ }^{53}$ This prove the correlation between enzymatic activities and the redox state in the HT22 cells exposure with glutamate. These results indicated the protection was mainly due to partial chemical structure of catechins and theaflavins, which contributes to regulate effect, rather than chemical binding to substrate.

Glutamate causes oxidative stress and excitotoxicity, however, HT22 cells lack functional ionotropic glutamate receptors, indicating neuronal cell death are triggered by oxidative stress. ${ }^{54-57}$ In this study, we shown that HT22 cells exposed to neurotoxic concentrations of glutamate undergo two distinct population: a subpopulation to necrosis during the exposure, while the other one to apoptosis. Apoptosis is a kind of programmed cell death. During this process, characteristic cell changes happen, including mitochondrial involved apoptotic pathway and several key enzymes. ${ }^{58}$ Our results showed that EGCG and TF3 protected HT22 cells against glutamate-induced cell apoptosis via regulation of cell apoptosis-related proteins, at least through the expression of Bcl-2 and Bax (Fig. 2 and 5E). EGCG and TF3 significantly increase the ratio of the two protein. Bcl-2 protein is similar to the nematode protein CED-9. Consistent with the role in blocking cell apoptosis. In contrast, Bax induces the release of cytochrome $\mathrm{C}$ and the induction of cell apoptosis. ${ }^{59}$ Cell necrosis was rarely protected by those two compounds (Fig. 2 and 5E). Notably, high concentrations of extracellular glutamate-induced cell death termed oxytosis/ferroptosis is distinct from apoptosis, necrosis. ${ }^{60}$ The high concentration glutamate exposure resulting effects on mitochondrial function are critical factors, as mitochondrial dysfunction is strongly associated with cell apoptosis. The findings are consistent with previous result and our recent observations (Fig. 4B). What is more, our data suggested that relatively intact mitochondrial activity of HT22 neuronal cell appears to be necessary for the apoptosis program to proceed. We found both catechins and theaflavins significantly reducing apoptosis induced by glutamate, a very encouraging result. The most prominent roles of mitochondria are participated and regulate cellular metabolism.

Moreover, the cell physiology was rescued by either catechins or theaflavins. Both those two families compounds affected $\mathrm{Ca}^{2+}$ influx, mitochondrial membrane potential, and also restored GSH related enzyme activity, and further decrease the ROS production (Fig. 3 and 4). Therefore, we concluded that the neuroprotective effect of theaflavins against glutamate-induced cell damage was also mediated through attenuation of oxidative stress. The repetitive monomer EGCG and its diarized product TF3 both effectively protected HT22 cells through changing their cell physiology.

We finally illustrate the protective mechanism of glutamateinduced HT22 cell damage. The results also show that EGCG and TF3-mediated ROS inhibits via AMPK and ERK phosphorylation (Fig. 5). Those signaling factors, regulate the progression of cell growth, proliferation and survival and phosphorylation of AMPK Thr172 strongly associates with cellular metabolic stress. ${ }^{61}$ On the other side, the present work had several limitations. Only partial regulators, AMPK and ERK phosphorylation were performed, meaning that the other ROS related pathways, including JNK, p38 are unknown. Thus, additional apoptosis proteins studies are required to assess the role of other pathways.

\section{Conclusion}

In summary, the current study demonstrated that anti-oxidative effect was found from both catechins and theaflavins. The typical representative compounds, EGCG and TF-3, restore glutamate-induced HT22 cell viability, reduce cell apoptosis by regulating Bax and Bcl-2 apoptosis proteins, attenuate ROS production via inverse correlation of AMPK and ERK phosphorylation. Theaflavins is an important natural source family of compounds, which reduces glutamate damage to neuronal cell. Therefore, the present study provides a novel potential therapeutic agent for glutamate accumulation and toxicity related diseases.

\section{Authors' contributions}

Jin-Ting He and Lei Xu performed the experiment; all author contributed to write the manuscript.

\section{Conflicts of interest}

The authors declare no conflicts of interest. 


\section{Acknowledgements}

The project supported by Jilin Provincial Department of Finance funds in China (No. Sczsy201512), Training Program Funds for Excellent Young Teachers in Jilin University (No. 419080500362) and Jilin Provincial Department of Health funds (No. 20152085).

\section{References}

1 Y. Zhou and N. C. Danbolt, Glutamate as a neurotransmitter in the healthy brain, J. Neural Transm., 2014, 121(8), 799-817.

2 C. Murphy-Royal, J. Dupuis, L. Groc and S. H. R. Oliet, Astroglial glutamate transporters in the brain: regulating neurotransmitter homeostasis and synaptic transmission, J. Neurosci. Res., 2017, 95(11), 2140-2151.

3 J. Preobraschenski, J. F. Zander, T. Suzuki, G. Ahnert-Hilger and R. Jahn, Vesicular glutamate transporters use flexible anion and cation binding sites for efficient accumulation of neurotransmitter, Neuron, 2014, 84(6), 1287-1301.

4 K. Moussawi, A. Riegel, S. Nair and P. W. Kalivas, Extracellular glutamate: functional compartments operate in different concentration ranges, Front. Syst. Neurosci., 2011, 5, 94.

5 D. A. Baker, Z. X. Xi, H. Shen, C. J. Swanson and P. W. Kalivas, The origin and neuronal function of in vivo nonsynaptic glutamate, J. Neurosci., 2002, 22(20), 9134-9141.

6 F. M. Ribeiro, L. B. Vieira, R. G. Pires, R. P. Olmo and S. S. Ferguson, Metabotropic glutamate receptors and neurodegenerative diseases, Pharmacol. Res., 2017, 115, 179-191.

7 V. Bruno, F. Caraci, A. Copani, F. Matrisciano, F. Nicoletti and G. Battaglia, The impact of metabotropic glutamate receptors into active neurodegenerative processes: a "dark side" in the development of new symptomatic treatments for neurologic and psychiatric disorders, Neuropharmacology, 2017, 115, 180-192.

8 N. Pregi, L. M. Belluscio, B. G. Berardino, D. S. Castillo and E. T. Canepa, Oxidative stress-induced CREB upregulation promotes DNA damage repair prior to neuronal cell death protection, Mol. Cell. Biochem., 2017, 425(1-2), 9-24.

9 H. Nakajima, M. Itakura, T. Kubo, A. Kaneshige, N. Harada, T. Izawa, et al., Glyceraldehyde-3-phosphate Dehydrogenase (GAPDH) Aggregation Causes Mitochondrial Dysfunction during Oxidative Stress-induced Cell Death, J. Biol. Chem., 2017, 292(11), 4727-4742.

10 F. Amri, I. Ghouili, M. Amri, A. Carrier and O. MasmoudiKouki, Neuroglobin protects astroglial cells from hydrogen peroxide-induced oxidative stress and apoptotic cell death, J. Neurochem., 2017, 140(1), 151-169.

11 H. S. Tiwari, U. K. Misra, J. Kalita, A. Mishra and S. Shukla, Oxidative stress and glutamate excitotoxicity contribute to apoptosis in cerebral venous sinus thrombosis, Neurochem. Int., 2016, 100, 91-96.

12 D. Colle, D. B. Santos, J. M. Hartwig, M. Godoi, D. F. Engel, A. F. de Bem, et al., Succinobucol, a Lipid-Lowering Drug, Protects Against 3-Nitropropionic Acid-Induced
Mitochondrial Dysfunction and Oxidative Stress in $\mathrm{SH}^{-}$ SY5Y Cells via Upregulation of Glutathione Levels and Glutamate Cysteine Ligase Activity, Mol. Neurobiol., 2016, 53(2), 1280-1295.

13 V. Singh, R. Gera, R. Kushwaha, A. K. Sharma, S. Patnaik and D. Ghosh, Hijacking microglial glutathione by inorganic arsenic impels bystander death of immature neurons through extracellular cystine/glutamate imbalance, Sci. Rep., 2016, 6, 30601.

14 Z. Li, X. Chen, W. Lu, S. Zhang, X. Guan, Z. Li, et al., AntiOxidative Stress Activity is Essential for Amanita caesarea Mediated Neuroprotection on Glutamate-Induced Apoptotic HT22 Cells and an Alzheimer's Disease Mouse Model, Int. J. Mol. Sci., 2017, 18(8), 1623.

15 P. K. Pan, L. Y. Qiao and X. N. Wen, Safranal prevents rotenone-induced oxidative stress and apoptosis in an in vitro model of Parkinson's disease through regulating Keap1/Nrf2 signaling pathway, Cell. Mol. Biol., 2016, 62(14), 11-17.

16 K. N. Prasad and S. C. Bondy, Inhibition of Early Biochemical Defects in Prodromal Huntington's Disease by Simultaneous Activation of Nrf2 and Elevation of Multiple Micronutrients, Curr. Aging Sci., 2016, 9(1), 61-70.

17 W. S. Sheng, S. Hu, A. Feng and R. B. Rock, Reactive oxygen species from human astrocytes induced functional impairment and oxidative damage, Neurochem. Res., 2013, 38(10), 2148-2159.

18 N. Shibata and M. Kobayashi, The role for oxidative stress in neurodegenerative diseases, Brain Nerve, 2008, 60(2), 157170.

19 D. Kong, H. C. Hu, E. Okuma, Y. Lee, H. S. Lee, S. Munemasa, et al., L-Met Activates Arabidopsis GLR $\mathrm{Ca}^{2+}$ Channels Upstream of ROS Production and Regulates Stomatal Movement, Cell Rep., 2016, 17(10), 2553-2561.

20 P. T. Schumacker, Reactive oxygen species in cancer: a dance with the devil, Cancer Cell, 2015, 27(2), 156-157.

21 Z. H. Ji, C. Liu, H. Zhao and X. Y. Yu, Neuroprotective effect of biatractylenolide against memory impairment in $\mathrm{D}$ galactose-induced aging mice, J. Mol. Neurosci., 2015, 55(3), 678-683.

22 M. Lalkovicova and V. Danielisova, Neuroprotection and antioxidants, Neural Regener. Res., 2016, 11(6), 865-874.

23 Y. Xu, H. Zhao, M. Zhang, C. J. Li, X. Z. Lin, J. Sheng, et al., Variations of antioxidant properties and NO scavenging abilities during fermentation of tea, Int. J. Mol. Sci., 2011, 12(7), 4574-4590.

24 C. Cao, T. Gao, M. Cheng, F. Xi, C. Zhao and W. Yu, Mild hypothermia ameliorates muscle wasting in septic rats associated with hypothalamic AMPK-induced autophagy and neuropeptides, Biochem. Biophys. Res. Commun., 2017, 490(3), 882-888.

25 J. Yang, L. Li, S. Tan, H. Jin, J. Qiu, Q. Mao, et al., A natural theaflavins preparation inhibits HIV-1 infection by targeting the entry step: potential applications for preventing HIV-1 infection, Fitoterapia, 2012, 83(2), 348-355. 
26 K. Yoshino, K. Yamazaki and M. Sano, Preventive effects of black tea theaflavins against mouse type IV allergy, J. Sci. Food Agric., 2010, 90(12), 1983-1987.

27 Y. Yang, Y. J. Qin, Y. W. Yip, K. P. Chan, K. O. Chu, W. K. Chu, et al., Green tea catechins are potent antioxidants that ameliorate sodium iodate-induced retinal degeneration in rats, Sci. Rep., 2016, 6, 29546.

28 S. Chikara, L. D. Nagaprashantha, J. Singhal, D. Horne, S. Awasthi and S. S. Singhal, Oxidative stress and dietary phytochemicals: role in cancer chemoprevention and treatment, Cancer Lett., 2018, 413, 122-134.

29 T. Cheng, J. Liu, J. Ren, F. Huang, H. Ou, Y. Ding, et al., Green Tea Catechin-Based Complex Micelles Combined with Doxorubicin to Overcome Cardiotoxicity and Multidrug Resistance, Theranostics, 2016, 6(9), 1277-1292.

30 S. Pournourmohammadi, M. Grimaldi, M. H. Stridh, V. Lavallard, H. S. Waagepetersen, C. B. Wollheim, et al., Epigallocatechin-3-gallate (EGCG) activates AMPK through the inhibition of glutamate dehydrogenase in muscle and pancreatic ss-cells: a potential beneficial effect in the prediabetic state?, Int. J. Biochem. Cell Biol., 2017, 88, 220-225.

31 T. Ye, J. Zhen, Y. Du, J. K. Zhou, A. Peng, N. D. Vaziri, et al., Green tea polyphenol (-)-epigallocatechin-3-gallate restores Nrf2 activity and ameliorates crescentic glomerulonephritis, PLoS One, 2015, 10(3), e0119543.

32 J. Yu, Y. Jia, Y. Guo, G. Chang, W. Duan, M. Sun, et al., Epigallocatechin-3-gallate protects motor neurons and regulates glutamate level, FEBS Lett., 2010, 584(13), 29212925.

33 C. T. Chu, D. J. Levinthal, S. M. Kulich, E. M. Chalovich and D. B. DeFranco, Oxidative neuronal injury. The dark side of ERK1/2, Eur. J. Biochem., 2004, 271(11), 2060-2066.

34 E. Runden, P. O. Seglen, F. M. Haug, O. P. Ottersen, T. Wieloch, M. Shamloo, et al., Regional selective neuronal degeneration after protein phosphatase inhibition in hippocampal slice cultures: evidence for a MAP kinasedependent mechanism, J. Neurosci., 1998, 18(18), 7296-7305.

35 J. T. Hwang, J. Ha, I. J. Park, S. K. Lee, H. W. Baik, Y. M. Kim, et al., Apoptotic effect of EGCG in HT-29 colon cancer cells via AMPK signal pathway, Cancer Lett., 2007, 247(1), 115121.

36 C. H. Huang, S. J. Tsai, Y. J. Wang, M. H. Pan, J. Y. Kao and T. D. Way, EGCG inhibits protein synthesis, lipogenesis, and cell cycle progression through activation of AMPK in p53 positive and negative human hepatoma cells, Mol. Nutr. Food Res., 2009, 53(9), 1156-1165.

37 H. Zhao, M. Zhang, L. Zhao, Y. K. Ge, J. Sheng and W. Shi, Changes of constituents and activity to apoptosis and cell cycle during fermentation of tea, Int. J. Mol. Sci., 2011, 12(3), 1862-1875.

38 H. Y. Lee, J. B. Weon, G. Ryu, W. S. Yang, N. Y. Kim, M. K. Kim, et al., Neuroprotective effect of Aronia melanocarpa extract against glutamate-induced oxidative stress in HT22 cells, BMC Complementary Altern. Med., 2017, 17(1), 207.
39 V. Adam-Vizi and A. A. Starkov, Calcium and mitochondrial reactive oxygen species generation: how to read the facts, $J$. Alzheimer's Dis., 2010, 20(suppl. 2), S413-S426.

40 A. Bajic, M. Spasic, P. R. Andjus, D. Savic, A. Parabucki, A. Nikolic-Kokic, et al., Fluctuating vs. continuous exposure to $\mathrm{H}_{2} \mathrm{O}_{2}$ : the effects on mitochondrial membrane potential, intracellular calcium, and NF-kappaB in astroglia, PLoS One, 2013, 8(10), e76383.

41 T. E. Tipple and L. K. Rogers, Methods for the determination of plasma or tissue glutathione levels, Methods Mol. Biol., 2012, 889, 315-324.

42 L. H. Chen, G. S. Zhu, D. L. Zhang, H. Zhao, M. Y. Guo, S. B. Wei, et al., Novel mesoporous silica spheres with ultra-large pore sizes and their application in protein separation, J. Mater. Chem., 2009, 19(14), 2013-2017.

43 M. Zhang, H. Zhao, X. Z. Shi, J. Wu, Q. Fu and W. Shi, Effects of DNAzymes and siRNA Targeting AKT1 on the Growth of Human T Leukemic Cells, Clin. Lab., 2014, 60(1), 1-8.

44 O. Weinreb, T. Amit, S. Mandel and M. B. H. Youdim, Neuroprotective molecular mechanisms of (-)-epigallocatechin-3-gallate: a reflective outcome of its antioxidant, iron chelating and neuritogenic properties, Genes Nutr., 2009, 4(4), 283-296.

45 X. Wang and M. G. Roper, Measurement of DCF fluorescence as a measure of reactive oxygen species in murine islets of Langerhans, Anal. Methods, 2014, 6(9), 3019-3024.

46 X. Y. Gao, S. N. Wang, X. H. Yang, W. J. Lan, Z. W. Chen, J. K. Chen, et al., Gartanin Protects Neurons against Glutamate-Induced Cell Death in HT22 Cells: Independence of Nrf-2 but Involvement of $\mathrm{HO}-1$ and AMPK, Neurochem. Res., 2016, 41(9), 2267-2277.

47 A. W. Siu, M. K. Lau, J. S. Cheng, C. K. Chow, W. C. Tam, K. K. Li, et al., Glutamate-induced retinal lipid and protein damage: the protective effects of catechin, Neurosci. Lett., 2008, 432(3), 193-197.

48 C. W. Chou, W. J. Huang, L. T. Tien and S. J. Wang, (-)-Epigallocatechin gallate, the most active polyphenolic catechin in green tea, presynaptically facilitates $\mathrm{Ca}^{2+}$ dependent glutamate release via activation of protein kinase C in rat cerebral cortex, Synapses, 2007, 61(11), 889902.

49 A. P. Lan, J. Chen, Z. F. Chai and Y. Hu, The neurotoxicity of iron, copper and cobalt in Parkinson's disease through ROSmediated mechanisms, BioMetals, 2016, 29(4), 665-678.

50 Y. Xiao, C. Karam, J. Yi, L. Zhang, X. Li, D. Yoon, et al., ROSrelated mitochondrial dysfunction in skeletal muscle of an ALS mouse model during the disease progression, Pharmacol. Res., 2018, 138, 25-36.

$51 \mathrm{~V}$. Roginsky and A. E. Alegria, Oxidation of tea extracts and tea catechins by molecular oxygen, J. Agric. Food Chem., 2005, 53(11), 4529-4535.

52 H. S. Kim, M. J. Quon and J. A. Kim, New insights into the mechanisms of polyphenols beyond antioxidant properties; lessons from the green tea polyphenol, epigallocatechin 3-gallate, Redox Biol., 2014, 2, 187-195.

53 M. S. Yang, H. W. Chan and L. C. Yu, Glutathione peroxidase and glutathione reductase activities are partially responsible 
for determining the susceptibility of cells to oxidative stress, Toxicology, 2006, 226(2-3), 126-130.

54 P. Jirasek, S. Amslinger and J. Heilmann, Synthesis of natural and non-natural curcuminoids and their neuroprotective activity against glutamate-induced oxidative stress in HT-22 cells, J. Nat. Prod., 2014, 77(10), 2206-2217.

55 E. Ramond, G. Gesbert, M. Rigard, J. Dairou, M. Dupuis, I. Dubail, et al., Glutamate utilization couples oxidative stress defense and the tricarboxylic acid cycle in Francisella phagosomal escape, PLoS Pathog., 2014, 10(1), e1003893.

56 E. J. Jeong, L. Hwang, M. Lee, K. Y. Lee, M. J. Ahn and S. H. Sung, Neuroprotective biflavonoids of Chamaecyparis obtusa leaves against glutamate-induced oxidative stress in HT22 hippocampal cells, Food Chem. Toxicol., 2014, 64, 397-402.
57 P. Maher and J. B. Davis, The role of monoamine metabolism in oxidative glutamate toxicity, J. Neurosci., 1996, 16(20), 6394-6401.

58 G. Morciano, G. Pedriali, L. Sbano, T. Iannitti, C. Giorgi and P. Pinton, Intersection of mitochondrial fission and fusion machinery with apoptotic pathways: Role of Mcl-1, Biol. Cell., 2016, 108(10), 279-293.

59 J. Pawlowski and A. S. Kraft, Bax-induced apoptotic cell death, Proc. Natl. Acad. Sci. U. S. A., 2000, 97(2), 529-531.

60 J. Lewerenz, G. Ates, A. Methner, M. Conrad and P. Maher, Oxytosis/Ferroptosis-(Re-) Emerging Roles for Oxidative Stress-Dependent Non-apoptotic Cell Death in Diseases of the Central Nervous System, Front. Neurosci., 2018, 12, 214.

61 K. H. Yan, C. J. Yao, C. H. Hsiao, K. H. Lin, Y. W. Lin, Y. C. Wen, et al., Mefloquine exerts anticancer activity in prostate cancer cells via ROS-mediated modulation of Akt, ERK, JNK and AMPK signaling, Oncol. Lett., 2013, 5(5), 1541-1545. 